\title{
Real-time Collision Risk Estimation based on Stochastic Reachability Spaces
}

\author{
Unmesh Patil, Alessandro Renzaglia, Anshul Paigwar and Christian Laugier
}

\begin{abstract}
Estimating the risk of collision with other road users is one of the most important modules to ensure safety in autonomous driving scenarios. In this paper, we propose new probabilistic models to obtain Stochastic Reachability Spaces for vehicles and pedestrians detected in the scene. We then exploit these probabilistic predictions of the roadusers' future positions, along with the expected ego-vehicle trajectory, to estimate the probability of collision risk in realtime. The proposed stochastic models only depend on the velocity, acceleration, tracked bounding box, and the class of the detected object. This information can easily be obtained through off-the-shelf 3D object detection frameworks. As a result, the proposed approach for collision risk estimation is widely applicable to a variety of autonomous vehicle platforms. To validate our approach, initially we test the stochastic motion prediction on the KITTI dataset. Further experiments in the CARLA simulator, by reproducing realistic collision scenarios, have the goal of demonstrating the effectiveness of the collision risk assessment and are compared with an alternative approach.
\end{abstract}

\section{INTRODUCTION}

To guarantee the safety of autonomous vehicles (AVs) and the robustness of driver assistance systems, it is essential to have a reliable evaluation of the risk of collision with the traffic participants. To estimate the risk, the system needs to perceive the environment, track the objects and predict their future actions. All the uncertainties involved in both sensing and prediction make this problem challenging. Achieving real-time, accurate detection and tracking of the objects is still an active field of research [1].

Given an accurate detection of the traffic participants in the scenes, a natural approach towards collision risk calculation is to predict their motion as oriented bounding boxes and continuously check their overlaps with the planned motion of the ego vehicle [2]. However, the motion of dynamic objects is subject to the involvement of human driver behavior, surrounding factors affecting the dynamics, and process noise. Moreover, the internal parameters such as weight, friction, etc. needed for the dynamic model are not observable by exteroceptive sensors, which demands the introduction of stochastic nature in the prediction [3].

After the prediction step, the risk needs to be quantified, but being an abstract term it is subjected to multiple definitions and formulations. Lefevre et al. have defined a broad classification of risk formulations in [4]. Collision is generally perceived as the main source of risk. However, other sources are also proposed, such as: unexpected behaviours, driver fatigue [5], and conflicting maneuver intentions [6].

The authors are with Univ Grenoble Alpes, Inria, 38000, Grenoble, France; e-mail: firstname. lastnamedinria.fr

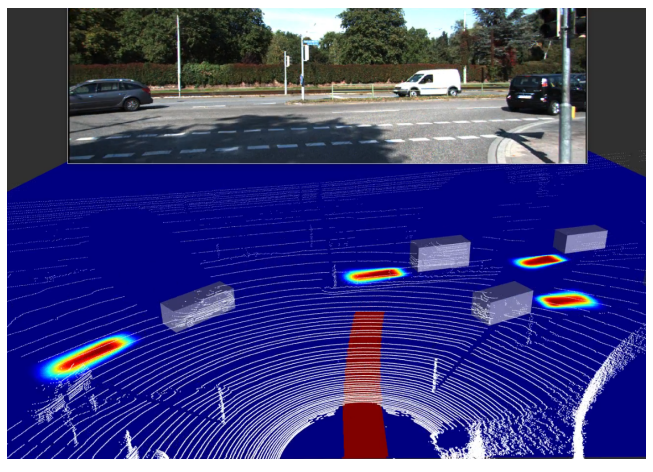

Fig. 1. An urban traffic scene from the KITTI dataset. The estimated swath of the ego vehicle (Red color) and the predicted occupancy distribution of traffic participants based on the proposed models is shown with color scale where red is the highest probability and blue is the lowest.

Multiple metrics are then considered to quantify the risk, e.g. Time-To-Collision (TTC), Time-To-Break, and TimeTo-React. Among all these sources and metrics, a widely adopted combination, also used in this work, is the risk of collision expressed in a probabilistic metric.

In this paper, we propose a new approach, based on stochastic models, to predict the future occupancy of vehicles and pedestrians (as shown in Fig. 1). Our models only require tracked bounding box details and the class of the object from object detection method like Frustum-pointpillars [7], while uncertainties in sensing and prediction are captured within the stochastic nature of the models. The main contributions of this work are summarised as follows:

1) We propose novel stochastic models to predict the future occupancy of vehicles and pedestrians.

2) Based on the predicted occupancy, we present a mapfree approach to estimate the probabilistic collision risk in real-time. Further, the approach is tested by creating multiple collision scenarios in the Carla simulator.

3) We perform experiments with the KITTI dataset to validate the prediction models. We compare the collision risk estimation with an alternative method to validate and compare the performance of our approach.

\section{RELATED WORK}

Motion prediction is a crucial aspect of collision risk estimation. Broadly, it can be classified into object-level prediction and sub-object or grid-based prediction. Object-level prediction can be divided into three classes: Reachability analysis, Monte-Carlo methods, and Learning-Based methods. In this work, we explore object-level motion prediction 
using reachability analysis.

In [8], Althoff et al. presented an approach adopting Markov chains and using kinematic models along with map data to obtain stochastic reachability sets for AVs. Map data can significantly improve the results in some scenarios but it limits the applications due to the frequent unavailability of detailed maps, format restrictions, and memory constraints. $\mathrm{Yu}$ et al. proposed a bi-directional reachability solution, taking into account sensory limitations and occlusions [9]. Stochastic Reachable (SR) sets are utilized in [10] to generate potential fields for moving obstacles. It is worth noticing that most of the existing methods for probabilistic risk estimation are computationally expensive, especially for cluttered traffic scenes. Monte-Carlo simulation methods are popular for probabilistic risk estimation, but such methods suffer from probabilistic errors due to the random sampling of the initial conditions and the input sequence as shown in [11].

The prediction of pedestrian's motion is even more challenging due to their larger available decision space. Among the several works proposing possible solutions to this problem, a recent paper presents very interesting results showing that a Constant Velocity Model (CVM) outperforms all existing state-of-the-art methods [12]. Our proposed approach to model pedestrian motion is in line with this work and a more detailed discussion is presented in Section III-B.

Contrary to object-based methods, grid-based methods predict occupancy and calculate risk for each cell obviating object detection and tracking. The Conditional Monte Carlo Dense Occupancy Tracker (CMCDOT) [13] belongs to this class of solutions. This approach is a spatial occupancy tracker that maps the environment into a probabilistic occupancy grid based on Bayesian fusion, filtering of sensor data, and Bayesian inference. particularly, it infers the dynamics of the scene via a hybrid representation, including static and dynamic occupancy, free spaces, and unknown regions. Following this work, Rummelhard et al. [14] estimated the future occupancy of particles in dynamically occupied cells to calculate TTC for each cell. This approach does not take into account the semantic information of objects for prediction and every cell is instead treated with the same model, producing less accurate results. Conversely, objectlevel risk estimation allows us to have object-specific motion models and semantic risk.

The proposed solution has the intent of overcoming the aforementioned limitations, incorporating a semanticdependent probabilistic collision risk estimation that can run in real-time, without the necessity of the map data and that it is widely applicable to many object detection frameworks.

\section{MOTION MODELS}

The first step in motion prediction is to design an appropriate motion model based on the estimated motion parameters. The main forces and moments affecting the motion of a car can be broadly classified into two categories: longitudinal and lateral. Firstly, in the longitudinal direction, the engine force, air drag, rolling friction, and momentum of the car. Secondly, in the lateral direction, opposite moments produced by tire forces, rotational inertia, and angular acceleration. The lateral motion can be described by the following simplified model:

$$
\ddot{\psi}=\frac{-c_{1} \beta}{I_{z}}-\frac{c_{2} \dot{\psi}}{I_{z} V}+\frac{c_{3} \delta}{I_{z}}=\frac{C \cdot \dot{\psi}}{V}+e
$$

where $\psi$ is the yaw angle, $\beta$ is side-slip angle, $V$ is the resultant velocity, and $I_{z}$ denotes the rotational inertia of the car. $c_{1}, c_{2}, c_{3}$ are constants dependent on the vehicle dimensions and cornering stiffness. The last expression highlights the proportionality of the angular acceleration to $\dot{\psi} / V$. A detailed derivation can be found in [15].

Deterministic dynamic vehicle models are used when motion parameters and driver intentions are perfectly known. However, such internal parameters of traffic participants are not observable by exteroceptive sensors. Also, deterministic models do not accommodate uncertainty resulting from human intentions. Such limitations make deterministic models unsuitable for reliable predictions. Instead, stochastic models are more appropriate to incorporate the uncertainties of realworld scenarios. With this purpose, we propose probability distributions for lateral and longitudinal components that contribute jointly to the final distribution, allowing separate and efficient handling of uncertainties.

\section{A. Stochastic Motion Models for Vehicle}

On the similar lines of longitudinal and lateral deterministic motion models discussed in the previous section, we propose the following radial and angular stochastic models to predict future positions of the vehicles in the scene.

1) Radial distribution: Let $D$ be the distance that a car will cover in a given time interval, and $u$ and $a$ be the current velocity and acceleration respectively. Hereafter, we indicate with the subscript actual the ground truth values while with the subscript $i$ the instantaneous values of motion parameters estimated as a difference between the last state and current state of the respective object. The instantaneous parameters cannot be used directly in future prediction given the uncertainties, hence we need to consider their deviation from actual values, i.e. $\Delta D=\left|D_{\text {actual }}-D_{i}\right|$. The evolution of $D_{i}$ and $D_{\text {actual }}$ over time can be expressed as follows:

$$
\begin{gathered}
D_{i}=u_{i} \cdot t+0.5 \cdot a_{i} \cdot t^{2} \\
D_{\text {actual }}=\left(u_{\text {actual }}\right) \cdot t+0.5 \cdot a_{\text {actual }} \cdot t^{2}
\end{gathered}
$$

Subtracting these two equations we then obtain:

$$
\Delta D=\Delta u_{i} \cdot t+0.5 \cdot \Delta a_{i} \cdot t^{2}
$$

$\Delta a_{i}$ and $\Delta u_{i}$ are the exact deviation values for a given instant. Considering the uncertainties involved, $D_{\text {actual }}$ is a stochastic variable, and we can approximate it as a distribution. To define such a distribution, we introduce a parabolic function $P$, with mean $\mu$ and support $\sigma_{k}$ that guarantees to respect the main requirements of having a high-probability zone close to the mean value and a fast decreasing likelihood near the support boundaries. The latter limits the spread of the probability tails in the state space avoiding to obtain a 

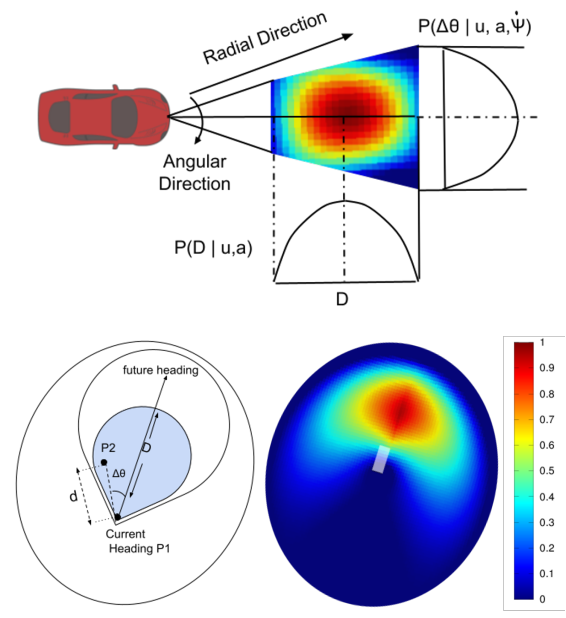

Fig. 2. Predicted probabilistic distributions using the proposed models for the motion of a vehicle (top) and a pedestrian (bottom). Distributions are scaled to the range shown in the legend for better visualisation.

too-conservative distribution that continuously flags unlikely collisions. The proposed distribution $P$ is:

$$
P\left(x \mid x_{0}\right)= \begin{cases}\frac{1}{K}\left(1-\frac{(x-\mu)^{2}}{\sigma_{k}}\right) & \text { if }(x-\mu)^{2} \leq \sigma_{k} \\ 0 & \text { if }(x-\mu)^{2}>\sigma_{k}\end{cases}
$$

where $K$ is a normalizing constant (the same is followed hereafter with relevant subscripts for all model equations). The model function $P$ is then used to define the longitudinal distribution. Let $D_{i}$ be the mean of all the possible distances that a car can cover given the initial conditions, and let $\Delta a_{\max }$ and $\Delta u_{\max }$ be the maximum possible deviations given the initial state. For any distance $D_{\text {actual }},(x-\mu)$ is equal to $\Delta D$ and the radial support $\sigma_{R}$ is equal to $\Delta u_{\max } \cdot t+\Delta a_{\max } \cdot t^{2} / 2$. Estimating the $\Delta u_{\max }$ and $\Delta a_{\max }$ is an important task. It has been stated before that it is impossible to accurately calculate the deviation but there are many possible ways to approximate it for a near-time horizon. The proposed expression for $\sigma_{R}$ as a function of the available instantaneous values, is:

$$
\sigma_{R}=\frac{1}{c_{f}}\left(u_{i} \cdot t \cdot \frac{u_{i}-1}{u_{i}+1}+\frac{a_{i} \cdot t^{2}}{2} \cdot \frac{a_{i}-1}{a_{i}+1}\right)
$$

where $c_{f}$ is a calibration factor depending on the class of the vehicle to account for its inertia. The calibration factor indicates the permissible extent of the deviation from kinematic expectation for a given class of vehicles (Truck, Car, Cyclist). The deviation term implies that, the motion in any future instant depends only on the instantaneous values of state variables. The multiplying factors $\left(u_{i}-1\right) /\left(u_{i}+1\right)$ and $\left(a_{i}-1\right) /\left(a_{i}+1\right)$ asymptotically fixate the upper bound on the support while regulating the extent of uncertainty based on the magnitude of the motion. The factor also limits the state variables to their maximum by modulating the likelihood. By using our proposed model, the resultant distribution, which is the likelihood that the vehicle would cover a given distance in a future instant, is:

$$
P\left(D_{\text {actual }} \mid u, a\right)=\frac{1}{K_{R}}\left(1-\frac{\Delta D^{2}}{\sigma_{R}}\right)
$$

Note that the above distribution is valid when $|a|>1$ and $|u|>1$. If these conditions are not met, then simple kinematic projections can be used.

2) Angular distribution: The angular distribution generates a likelihood of Centre of Mass (COM) in the plane, in terms of angular deviation from the current position. Let the difference between the current heading and the next heading be $\Delta \theta$. Ideally, for a constant yaw-rate system the value of $\Delta \theta$ should be equal to $\dot{\psi} t$. However, the human involved in the steering action can change the angular velocity and hence the difference between actual $\Delta \theta$ and $\dot{\psi} t$ has to be expressed as a probabilistic distribution.

From the model in eq. (1), the angular acceleration is proportional to $\dot{\psi} / V$. We express the instantaneous angular acceleration as a difference between the actual angular velocity at a given instant and angular velocity estimated from previous instant. With the help of eq. (1) we obtain:

$$
(\Delta \theta-\dot{\psi} t)=\left(C \dot{\psi} t^{2}\right) / V+e
$$

where $e$, defined in eq. (1), can be interpreted as an error term. Similar to the radial distribution, we propose an angular distribution for the stochastic variable $\Delta \theta$, with mean $\dot{\psi} t$ and angular support $\sigma_{A}$, defined using eq. (8):

$$
P(\Delta \theta \mid u, a, \dot{\psi})=\frac{1}{K_{A}}\left(1-\frac{(\Delta \theta-\dot{\psi} t)^{2}}{\sigma_{A}}\right)
$$

The resultant distribution as a product of the radial and the angular distribution is shown in Fig 2.

\section{B. Stochastic Motion Model for Pedestrian}

Apart from vehicles, pedestrians are also major participants in traffic scenes. Their motion is highly unpredictable but some general assumptions can still be made: 1) In a normal scenario, pedestrians will seldom make a 180-degree turn instantly and straight lines can be usually assumed to connect two close points. 2) The general bounds for a pedestrian motion, as reported in [16], are: average reach distance $=2 \mathrm{~m}$, maximum acceleration $=2 \mathrm{~m} / \mathrm{s}^{2}$, maximum velocity $=3.33 \mathrm{~m} / \mathrm{s}$. Considering these observations, we propose the following radial and angular models:

$$
\begin{gathered}
P(d \mid D)=\frac{1}{K_{P}}\left(1-\frac{(d-D)^{2}}{D_{\max }}\right) \\
P\left(\Delta \theta \mid \theta_{t}\right)=\frac{1}{K_{N}}\left(1-\left|\sin \left(\frac{\Delta \theta}{2}\right)\right|\right)
\end{gathered}
$$

with $\Delta \theta=\left(\theta_{t+i}-\theta_{t}\right)$. For a given point in space, $d$ is the distance from the pedestrian's current location and $D$ is the expected distance to be covered by pedestrian for a short time horizon based on CVM. $D_{\max }$ is the maximum reach of a pedestrian for a given time horizon. The angular model is based only on the current orientation. The radial model is instead in accord with the parabolic distribution in eq. (5), 


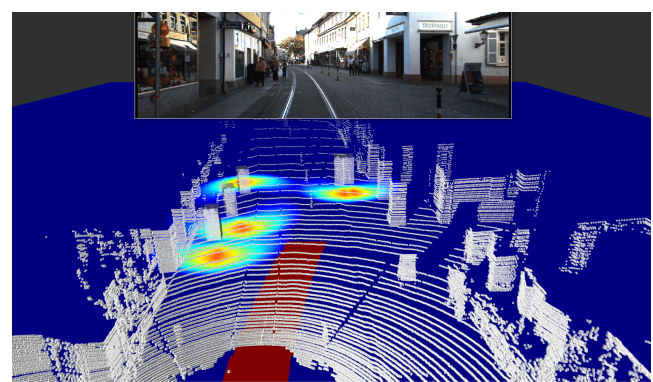

Fig. 3. A narrow lane urban scene with multiple pedestrians from KITTI raw data. The estimated swath of the ego vehicle is shown in red. The predicted occupancy distributions of traffic participants are depicted with a color scheme for the given time horizon.

where the mean is the distance calculated by CVM and the deviation is the maximum reach. An illustrative example of the final distribution is shown in Fig. 2, while Fig. 3 shows an instance of the predicted occupancy for pedestrians using the proposed model in a real-world scenario.

\section{COLLISION Risk Estimation}

To obtain the probability of collision, we firstly compute the SR sets for every participant in traffic to predict its future occupancy. The previously defined motion models generate a continuous normalized distribution of predicted COMs of the object. However, these distributions do not consider the dimensions of the object and cannot be used directly for collision risk estimation. Hence the motion models are used to first create SR sets and then the collision risk is evaluated. To create SR sets, the orientation for every reachable COM is mandatory along with dimensions. Dimensions are retrieved from bounding box details and the orientation is estimated by assuming circular trajectories as explained below.

Let $C$ be the set of all possible positions in a discrete space that can be achieved by the COM of vehicle in the future instant, i.e. $C=\left\{x_{i} \mid P\left(x_{i} \mid x_{0}\right)>0\right\}$, where $P(x)$ is the product of radial and angular probabilities for a given state $x$. For any of these points, we associate the corresponding orientation. Let $\left(x_{1}, y_{1}\right)$ be the initial position and $\left(x_{2}, y_{2}\right)$ a point in $C$, the angle $T$ that indicates the orientation of the vehicle is:

$$
T=2 \arctan \left(\frac{y_{2}-y_{1}}{x_{2}-x_{1}}\right)
$$

Oriented rectangles are then generated for each point in $C$, based on the vehicle orientation and dimension. We define a box operator which returns all points in the oriented rectangle for a given $\mathrm{COM}$, and any point within a given rectangle shares the same probability of the corresponding COM. By summing up the contributions coming from all the possible overlapping oriented rectangles, we obtain the final probabilities of occupancy for the entire map, as shown in Fig 4. Formally, we can express the probability that a given point $x$ will be occupied as:

$$
p(x)=\sum_{x_{i} \in \Omega(x)} P\left(x_{i}\right)
$$

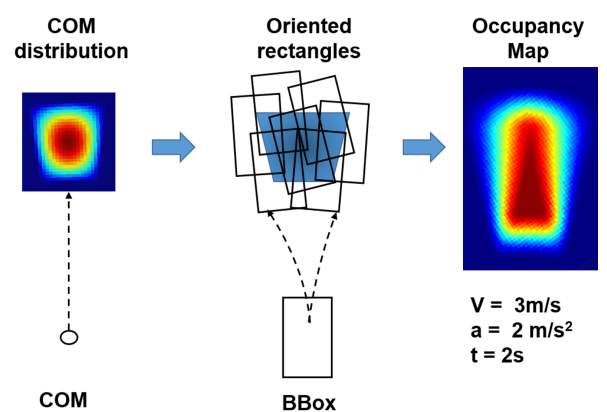

Fig. 4. Generation of occupancy map from predicted distribution of COM. An oriented rectangle is generated for each point in the COM distribution and then the probabilities are combined to create the final occupancy map.
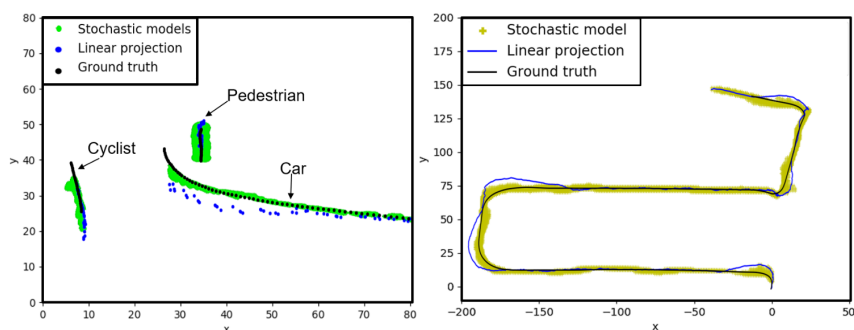

Fig. 5. Left: qualitative validation of motion models by comparing the prediction (green) with ground truth (black). The results of an instantaneous linear projection (blue) for the same time horizon of 2 seconds are also shown. Right: representative example of motion prediction considering the ego-vehicle motion over a $500 \mathrm{~m}$ trajectory in the KITTI raw data with a $3 s$ time horizon. The predicted COM positions, the ground truth and the linear projection are shown in green, black and blue respectively.

where,

$$
\Omega(x)=\left\{x_{i} \mid x \in \operatorname{box}\left(x_{i}\right)\right\} .
$$

We can then define the occupancy set of a given object $H$ as the set of points with a non-zero probability to be occupied, i.e. $H=\{x \mid p(x)>0\}$. Finally, let $S$ be the set of points belonging to the swath of the ego vehicle, the collision risk $R$ is given by:

$$
R=\max _{x_{j} \in(H \cap S)} p\left(x_{j}\right) .
$$

\section{EXPERIMENTS \& RESULTS}

In this section, we present the results obtained to validate the proposed approach. All experiments have been carried on an Intel Core i5 CPU $2.3 \mathrm{GHz}, 8 \mathrm{~GB}$ RAM, using NUMBA parallelization feature. The average time taken for each iteration of the proposed algorithm is around $14 \mathrm{~ms}$ for a grid resolution of $0.1 \mathrm{~m}$.

\section{A. Validation of motion models}

To analyze the performance of the proposed stochastic motion prediction models, we first consider scenes from the KITTI raw data wherein we have multiple types of dynamic objects. One such scene is shown in Fig. 5 (Left), where we have dynamic Pedestrian, Cyclist, and Car. The motion of all three types of objects is predicted simultaneously and plotted against ground truth (GT) and linear projection (LP). The values of the parameters for all the experiments here 
TABLE I

TABLE1: RESUlTS ON THE KITTI DATASET.

\begin{tabular}{|c|ccc|}
\hline & \multicolumn{3}{|c|}{ FDE $(m)$} \\
Method & $1 \mathrm{~s}$ & $2 \mathrm{~s}$ & $3 \mathrm{~s}$ \\
\hline Kalman filter & 0.46 & 1.18 & 2.18 \\
Linear Regression & 0.47 & 1.13 & 1.94 \\
RS $(P>0.99)$ & 0.33 & 0.75 & 1.23 \\
RS $(P>0.95)$ & 0.31 & 0.69 & 1.02 \\
RS $(P>0.9)$ & $\mathbf{0 . 3 1}$ & $\mathbf{0 . 6 4}$ & $\mathbf{0 . 9 4}$ \\
\hline
\end{tabular}

presented are: 1) In eq. (6), $c_{f}=2.08$ for cars and $c_{f}=2.30$ for cyclist; 2) In eq. (8), $C=0.14$ for all vehicles. All parameters have been calibrated using two sequences for each class of object from the KITTI dataset by employing an exhaustive search in the parameter space for each model.

Since in the KITTI dataset obstacles appear only for a short interval in the observation frame, it is impossible to evaluate the prediction results for a long time. To overcome this problem, we consider the ego vehicle itself as the object of our prediction. We first transform the GPS coordinates to the map frame and then estimate instantaneous state variables to predict the future state for a fixed time horizon. We perform prediction experiments on KITTI raw data and evaluate our results. For evaluation we use the Final Displacement Error (FDE) as a metric. This metric calculates the Euclidean distance between the predicted and the true final destination for the given time horizon. Given the stochastic nature of the distribution, FDE is not an ideal metric. However, the main intention of using this metric is to have a first evaluation of how close is the peak region to the ground truth. To obtain the FDE, we firstly consider a high probability region, defined as the set of all points having a probability greater than a threshold value $P_{\text {threshold }}$. Then, we evaluate the average FDE over the high probability region. It is important to remark that for the final collision risk evaluation, the complete distribution is considered and not only this high probability region. Average FDEs evaluated over the KITTI dataset for multiple $P_{\text {threshold }}$ values ( $\left.P_{\text {threshold }}=0.9,0.95,0.99\right)$ are shown in Table I. One such sample sequence from the dataset is plotted in Fig. 5 (Right). Also, we compare our approach with two standard baselines: $i$ ) a Kalman filter, with a constant speed model used to propagate the estimate without considering measures; ii) a linear coordinate regression method.

\section{B. Effect of state variables}

In this subsection, we analyze how the predicted future occupancy of vehicles varies with state variables. State variables contribute jointly to the final occupancy map and, since the uncertainties are handled separately, observing the combined effect is important for qualitative analysis. From Fig. 6, it can be observed how the uncertainty grows with the velocity for cases 1 to 3 . As the velocity increases the angular spread of distribution decreases, which is in line with the inertial properties of the car and the usual behavior of drivers. For cases 4 and 5, the growing uncertainty in the lateral direction is observed due to the angular velocity.

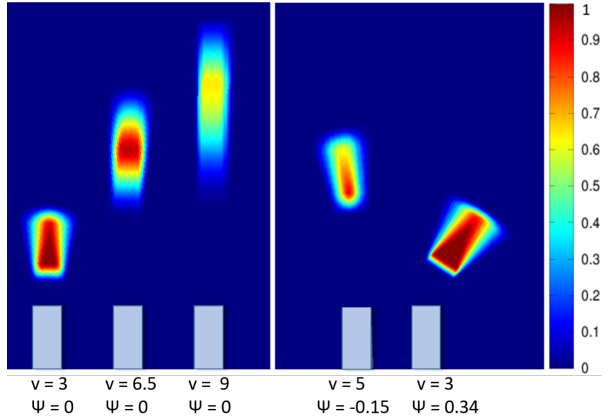

Fig. 6. Some of the possible resultant distributions and the corresponding values of state variables in SI units. Case numbers 1 to 5 from left to right.

TABLE II

COLLISION RISK EVALUATION (3S TIME HORIZON).

\begin{tabular}{|c|c|}
\hline Scenarios & Avg. decision window \\
\hline Collision with a leading vehicle & $2.83 \mathrm{~s}$ \\
Pedestrian crossing & $1.61 \mathrm{~s}$ \\
Collision at a lane merge & $2.92 \mathrm{~s}$ \\
Collision while overtaking & $2.45 \mathrm{~s}$ \\
head-on collision & $2.89 \mathrm{~s}$ \\
\hline
\end{tabular}

\section{Validation of collision risk}

Considering the final collision risk estimation, we test our results by creating several collision scenarios in the CARLA simulator with the help of the Scenario-runner and Carla ROS-Bridge, as the one shown in Fig. 7. For more information on the scenarios generation and their variability please refer to [17]. The results are then compared with the collision risk generated by the previously described CMCDOT method [14]. We here provide the results for a scenario where two cars coming from perpendicular directions collide at the junction. The ego vehicle is keeping a constant velocity while the other vehicle is accelerating towards the junction at $70 \%$ of the maximum acceleration. In Fig. 8, the predicted risk is plotted against time for both CMCDOT and the proposed collision risk estimation method for the $3 \mathrm{~s}$ time horizon. The Euclidean distance between the two cars is plotted below each plot to indicate the GT. The horizontal blue line indicates a risk threshold at 0.3 to start flagging the collision. The collision happens exactly at $6.18 \mathrm{~s}$. The threshold value is selected as a trade-off between frequent false collision reports and delay in collision prediction, after observing experiments on both CMCDOT and our method. For the proposed method, the collision is flagged at about three seconds before the collision happened, allowing a 2.95seconds decision window for the autonomous driver to take necessary actions. The CMCDOT failed to flag the collision on time and produced only a $1.75 \mathrm{~s}$ decision window. The proposed method showed early detection and produced a $76 \%$ larger decision window for this representative example.

Multiple scenarios created in the Carla simulator (see Fig. 9 and video attachment) are used to evaluate the proposed method on the basis of the decision window. Results are reported in Table II. 

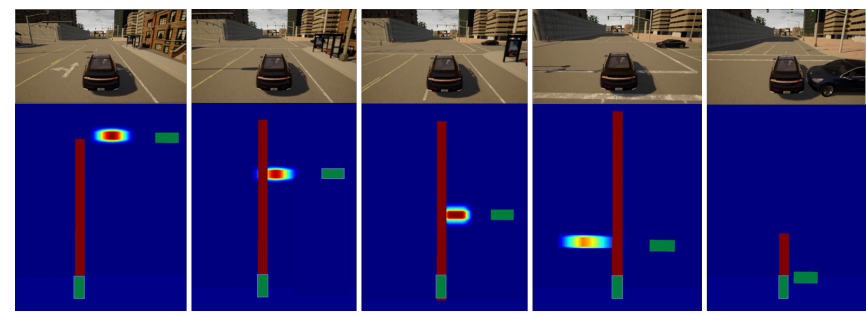

Fig. 7. A collision scenario at a road junction in CARLA simulator is presented along with the corresponding occupancy prediction for collision risk estimation. The red rectangle represents the swath of the ego vehicle. The bounding boxes of both the vehicles are shown in green.

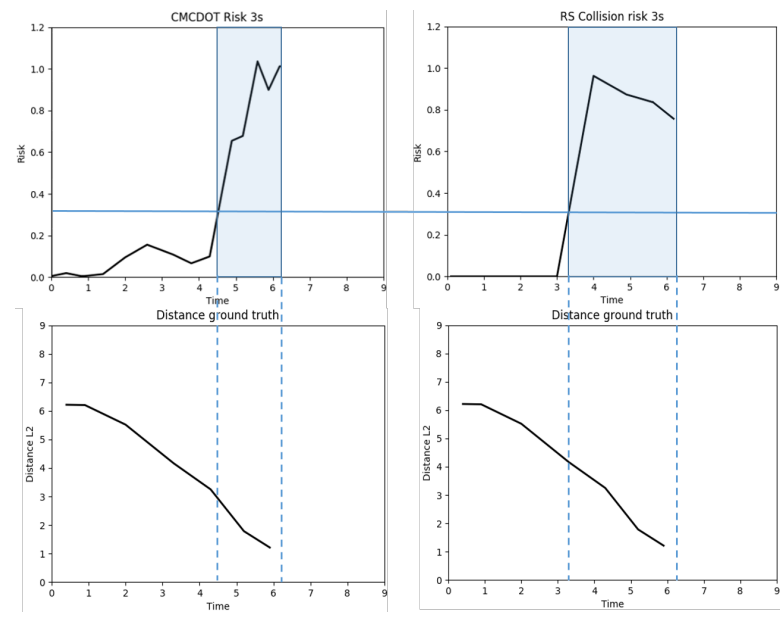

Fig. 8. Comparison between the collision risk generated by the CMCDOT and the proposed approach. The rectangles highlighted in blue show the decision window generated by each approach or time available to react and avoid the collision. The ground truth distance between two vehicles is plotted below both risk plots to mark the time stamp of collision. The decrease in the risk value is the result of the fleeting time horizon.

\section{CONCLUSIONS}

In this paper, we tackled the problem of estimating the risk of collision with dynamic objects in autonomous driving scenarios. We presented a solution based on stochastic reachability spaces to provide a probabilistic prediction of the motion of objects in the scenes. The proposed distributions depend on the class of the detected objects to incorporate specific motion-prediction models. The resulting approach has the advantage of being map-free and having limited computational requirements, allowing real-time performance. Extensive experimental results on both the KITTI dataset and recreating collision events in the CARLA simulator proved the performance of this approach. Our method detected collisions accurately, allowing a wide decision window for autonomous driving even during highly dynamic accelerated motion. The predicted risk is also compared with the existing CMCDOT approach showing a significant improvement.

In the future, we intend to add more contextual information of the scene to the models by exploiting vision-based methods to estimate road structure in real-time. We also plan to combine the current approach with a detection and classification module to handle the uncertainties arising from false detection or misclassification.

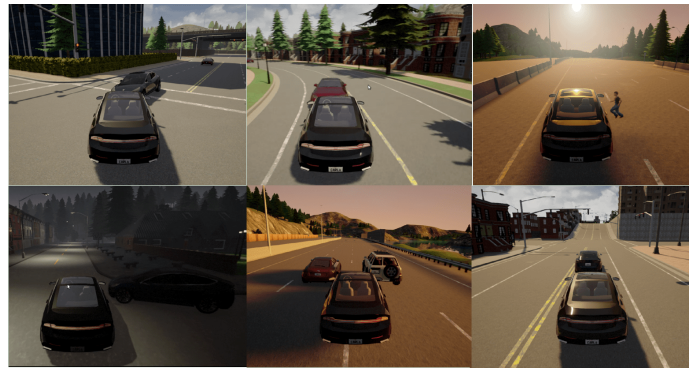

Fig. 9. Collision scenarios created in Carla simulator.

\section{REFERENCES}

[1] R. Verma, "A review of object detection and tracking methods," International Journal of Advance Engineering and Research Development, vol. 4, pp. 569-578, 102017.

[2] A. Philipp and D. Goehring, "Analytic collision risk calculation for autonomous vehicle navigation," in 2019 International Conference on Robotics and Automation (ICRA). IEEE, 2019, pp. 1744-1750.

[3] N. Deo, A. Rangesh, and M. M. Trivedi, "How would surround vehicles move? a unified framework for maneuver classification and motion prediction," IEEE Transactions on Intelligent Vehicles, vol. 3 , no. 2, pp. 129-140, 2018.

[4] S. Lefèvre, D. Vasquez, and C. Laugier, "A survey on motion prediction and risk assessment for intelligent vehicles," ROBOMECH journal, vol. 1, no. 1, pp. 1-14, 2014.

[5] S. Worrall, D. Orchansky, F. Masson, and E. Nebot, "Improving vehicle safety using context based detection of risk," in 13th IEEE International Conference on Intelligent Transportation Systems, 2010.

[6] S. Lefèvre, C. Laugier, and J. Ibañez-Guzmán, "Evaluating risk at road intersections by detecting conflicting intentions," in 2012 IEEE/RSJ International Conference on Intelligent Robots and Systems. IEEE, 2012, pp. 4841-4846.

[7] A. Paigwar, D. Sierra-Gonzalez, O. Erkent, and C. Laugier, "Frustumpointpillars: A multi-stage approach for $3 \mathrm{~d}$ object detection using rgb camera and lidar," in Proceedings of the IEEE/CVF International Conference on Computer Vision, 2021, pp. 2926-2933.

[8] M. Althoff, O. Stursberg, and M. Buss, "Model-based probabilistic collision detection in autonomous driving," IEEE Transactions on Intelligent Transportation Systems, vol. 10, no. 2, pp. 299-310, 2009.

[9] M. Y. Yu, R. Vasudevan, and M. Johnson-Roberson, "Risk assessment and planning with bidirectional reachability for autonomous driving," in 2020 IEEE International Conference on Robotics and Automation (ICRA), 2020, pp. 5363-5369.

[10] N. Malone, H. T. Chiang, K. Lesser, M. Oishi, and L. Tapia, "Hybrid dynamic moving obstacle avoidance using a stochastic reachable setbased potential field," IEEE Transactions on Robotics, vol. 33, no. 5, pp. 1124-1138, 2017.

[11] M. Althoff and A. Mergel, "Comparison of markov chain abstraction and monte carlo simulation for the safety assessment of autonomous cars," IEEE Transactions on Intelligent Transportation Systems, vol. 12, pp. 1237-1247, 2011.

[12] C. Schöller, V. Aravantinos, F. Lay, and A. Knoll, "What the constant velocity model can teach us about pedestrian motion prediction," IEEE Robotics and Automation Letters, vol. 5, no. 2, pp. 1696-1703, 2020.

[13] L. Rummelhard, A. Negre, and C. Laugier, "Conditional monte carlo dense occupancy tracker," in 2015 IEEE 18th International Conference on Intelligent Transportation Systems, 2015, pp. 2485-2490.

[14] L. Rummelhard, A. Nègre, M. Perrollaz, and C. Laugier, "Probabilistic grid-based collision risk prediction for driving application," in Experimental Robotics. Springer, 2016, pp. 821-834.

[15] M. Althoff and J. M. Dolan, "Online verification of automated road vehicles using reachability analysis," IEEE Transactions on Robotics, vol. 30, no. 4, pp. 903-918, 2014.

[16] M. Hartmann and D. Watzenig, "Pedestrians walking on reachable sets and manifolds," 2019 IEEE International Conference on Mechatronics (ICM), vol. 1, pp. 562-569, 2019.

[17] A. Paigwar, E. Baranov, A. Renzaglia, C. Laugier, and A. Legay, "Probabilistic collision risk estimation for autonomous driving: Validation via statistical model checking," in IEEE Intelligent Vehicles Symposium (IV), 2020, pp. 737-743. 